\title{
Evaluación del cumplimiento de la guía en salud mental para atención de duelo perinatal en el ámbito hospitalario
}

\section{Evaluation of compliance of the mental health guide for perinatal grief care in the hospital setting}

\author{
María Esperanza Durán-Flórez ${ }^{1}$ y Diana Marcela Casallas-Murillo² \\ Citación: Durán F., M.E. y Casallas M., D.M. (2019). Evaluación del cumplimiento de la guía en salud \\ mental para atención de duelo perinatal en el ámbito hospitalario. Psicología y Salud, 29(1), 91-102.
}

\section{RESUMEN}

\begin{abstract}
La muerte perinatal representa el inicio de un proceso de duelo que afecta las esferas física, emocional y social del núcleo familiar, principalmente de los padres. Objetivo: Evaluar el cumplimiento de las fases que componen la guía de atención de duelo perinatal en el ámbito hospitalario a través de una lista de chequeo y una entrevista a las madres en duelo, teniendo en cuenta la percepción subjetiva de las mismas respecto a cada fase de la atención brindada. Materiales y método: En este estudio exploratorio, prospectivo, analítico y de tipo mixto se obtuvo una muestra de nueve pacientes de la Clínica Universitaria Colombia, quienes fueron atendidas por duelo perinatal. Se evaluó el cumplimiento de la guía mediante una lista de chequeo como instrumento de verificación durante el proceso; además, por medio de una entrevista semiestructurada, se tuvieron en cuenta las percepciones de las pacientes sobre el proceso de apoyo brindado en el ámbito hospitalario por los profesionales tratantes. Resultados: Se llevó a cabo un análisis cualitativo y otro cuantitativo, de acuerdo con las categorías establecidas, con la finalidad de proponer recomendaciones para el manejo de esta población que pudiesen facilitar ajustes posteriores de la citada guía. Discusión: Es importante llevar a cabo procesos de evaluación de la atención hospitalaria en conjunto con las pacientes y los profesionales de la salud, lo que conduce a lograr aportaciones positivas a la retroalimentación y el mejoramiento continuo en un problema tan sensible como el duelo perinatal.
\end{abstract}

Palabras clave: Guía; Salud mental; Duelo; Atención perinatal.

\begin{abstract}
Perinatal death represents the beginning of a grieving process which impacts on the physical, emotional and social spheres of the family nucleus, specially the parents. Objective: To evaluate the compliance with the phases that make up the perinatal grief care guide in the hospital environment, through a checklist and interview to bereaved mothers, including the subjective perception of women, with respect to each phase of care provided. Materials and method: It is an exploratory, prospective, analytical, mixed type study. Participants were nine patients obtained from the University Clinic Colombia, treated between June and August 2016 for perinatal grief. The evaluation of compliance with the guide was carried out; using a checklist as a verification tool during the process. In addition, a semi-structured interview collected perceptions of patients of the support process provided in the hospital professionals. Results: Qualitative and quantitative analysis of categories, led to proposing recommendations in the management of this population, which may in turn contribute to adjustments for the guide. Conclusion: It is important to carry out evaluation processes on hospital care, in conjunction with patients and health professionals, which generates positive feedback and continuous improvement in a subject as sensitive as perinatal grief.
\end{abstract}

Keywords: Guide; Mental health; Grief; Perinatal care.

\footnotetext{
${ }^{1}$ Clínica Universitaria Colombia, Calle 22B, Núm. 66-46, Bogotá, Colombia, tel. (57)31-25-88-04-79, correo electrónico: meduran@colsanitas.com. Artículo recibido el 15 de enero y aceptado el 7 de junio de 2018.

${ }^{2}$ Universidad El Bosque, No 131 A, Ak. 9 \# 131 a 2, Bogotá, Colombia, tel. (57)30-07-81-45-00, correo electrónico: dmcasallas@unbosque.edu.co.
} 


\section{INTRODUCCIÓN}

$\mathrm{L}$ a maternidad es un constructo mediado por creencias y factores socioculturales de la persona, de su núcleo más cercano y de la sociedad, por lo que se convierte en un concepto que varía constantemente dependiendo del tiempo, el lugar y el contexto en que ocurre; por ello, la mujer embarazada tiene diferentes concepciones de su situación a partir de representaciones de carácter personal y experiencias individuales, lo que le hace posible estar vinculada en mayor o menor grado con el feto (Bello, 2016).

A lo anterior se suma la construcción de ideales sobre el futuro bebé a través de cambios que responderán a los nuevos planes y roles, generando la relación entre los padres y el hijo durante el periodo intrauterino, por lo que cualquier impacto negativo durante el proceso del embarazo afectará la salud emocional del núcleo familiar, especialmente de la madre (García y Yagüe, 1999).

De acuerdo con las condiciones médicas de la mujer en estado de embarazo, es posible iniciar tratamientos enfocados a estabilizarla, procurando así la supervivencia del feto; sin embargo, en ciertas situaciones tales intervenciones médicas no podrán cumplir el objetivo, por lo que, acto seguido, ocurrirá la muerte del feto o el neonato; es en estos momentos, cuando los familiares afectados tienen reacciones de profundo dolor, las que en ocasiones pueden llegar a ser un evento traumático que se acompaña de otros tipos de emociones de difícil manejo (Gold, Dalton y Schwenk, 2007).

Las muertes ocurridas durante los periodos fetal y neonatal se clasifican según criterios clínicos internacionales, de tal manera que en el primer periodo se considera la muerte fetal, que incluye abortos -que son pérdidas fetales de peso menor de 500g hasta la vigésimo-segunda semana de gestación- y mortinatos, con peso mayor a $500 \mathrm{~g}$ y que ocurren después de la referida semana de gestación. Además, se encuentran las muertes neonatales, que a su vez se dividen en muertes neonatales tempranas, que se producen desde el nacimiento hasta los primeros siete días, y muertes neonatales tardías, que van desde los siete hasta los veintiocho días luego del nacimiento. En con- junto, engloban las muertes perinatales (Instituto Nacional de Salud Pública, 2016).

De acuerdo con cifras entregadas o reportadas por la Organización Mundial de la Salud (OMS) (2015a), en el año 2015 ocurrieron 303,000 muertes maternas en el mundo asociadas a complicaciones en la gestación; 2.7 millones de neonatos fallecieron durante el primer mes de vida, y cifras similares corresponden a mortinatos. En relación al panorama nacional, según la Encuesta Nacional de Demografía y Salud del Departamento Administrativo Nacional de Estadística (DANE) (2016a, 2016b), en ese mismo año hubo 4,364 muertes neonatales y 46,129 muertes fetales, esto es, 49,116 muertes perinatales. Lo anterior pone de manifiesto que la cifra de padres afectados emocionalmente por la muerte de sus hijos representa cerca del doble del número de muertes perinatales, sin contar con otros familiares, como los abuelos o hermanos, que de igual forma atraviesan por el duelo.

Según la Organización Panamericana de la Salud (OPS) (2016), el duelo es un proceso que involucra factores emocionales que se hacen presentes luego de una pérdida; dichas reacciones remiten generalmente, sin que sean consideradas como un trastorno psicológico, si bien son las personas con síntomas intensos quienes acuden con mayor frecuencia a los servicios de salud para recibir atención mental. Derivado de lo anterior, se describe al duelo como un fenómeno "normal", no asociado en principio a patologías, aunque puede convertirse en un duelo "complicado" si se torna permanente o surgen durante éste alteraciones psicopatológicas de gran intensidad (Medina, 2015). Según los criterios diagnósticos del DSM-V (American Psychiatric Association, 2013), dichas dificultades en el proceso de adaptación a la pérdida tienen una prevalencia de 2.4 a $4.8 \%$, con una ocurrencia mayor en las mujeres, quienes generalmente están en riesgo de desarrollar un trastorno de duelo complejo y persistente.

En consecuencia, el duelo implica una mayor susceptibilidad de los familiares afectados para sufrir enfermedades físicas y mentales, además de una mayor vulnerabilidad a morir entre los padres luego de la pérdida ( $\mathrm{Li}$, Precht, Mortensen y Olsen, 2003). 
Para evaluar algunos aspectos relacionados con la intensidad del duelo perinatal, Hutti et al. (2018) validaron una escala que permite diagnosticar síntomas depresivos y ansiedad luego de tres meses desde la pérdida. Este tipo de instrumentos ayudan a los equipos de salud a detectar mujeres en duelo perinatal que requieran la intervención en salud mental para controlar riesgos emocionales severos.

Al ocurrir la muerte perinatal o neonatal, es posible que los padres manifiesten sentimientos de culpa, frustración, ideas de injusticia o castigo, y en el largo plazo complicaciones que pueden desencadenar enfermedades psicológicas y emocionales, con un mayor riesgo durante los primeros dos años después de la pérdida. Dichos síntomas pueden estar presentes no solo en los padres, sino también en otros integrantes de la familia o en personas cercanas (Oviedo, Urdaneta, Parra y Marquina, 2009).

Aunque la reacción frente a una pérdida es única y se vivencia de manera distinta en virtud de que las sensaciones que la acompañan son personales e intransferibles, se han encontrado reacciones similares en el momento en que ocurre la muerte perinatal, cuando en el primer momento son generalmente los padres quienes sufren crisis como respuesta a la ruptura homeostática al no disponer de mecanismos de afrontamiento que generen una respuesta oportuna y la restauración del equilibrio, lo que a su vez tiene resultados conductuales, emocionales, cognitivos y biológicos (Rabelo, 2010). Es entonces cuando debe comenzar la intervención en salud mental dentro del ambiente hospitalario, que en el primer momento está a cargo de médicos, parteras profesionales o enfermeras, pues de ellos depende la atención ginecobstétrica de emergencia durante el embarazo y el posparto y su consecuente manejo, así como, en caso de presentarse complicaciones en la salud materna, la remisión a otros profesionales (Fescina, De Mucio, Ortiz y Jarquín, 2012).

El tratamiento posterior al egreso generalmente no incluye la atención psicológica (Geller, Psaros y Kornfield, 2010). Boelen, Keijser, van den Hout y van den Bout (2007) indican que dichas intervenciones dirigidas a los problemas relacionados con el duelo tienen efectos benéficos sobre la tristeza y la preocupación, sobre todo si se realizan poco tiempo después de ocurrida la pérdida (cf. Sanz, García y Carbajo, 2014). No obstante que la OPS (2011) recomienda que las acciones en el área de la salud mental deben estar principalmente destinadas a la atención temprana de psicopatologías o desórdenes adaptativos que puedan afectar el bienestar y calidad de vida de pacientes en la etapa de gestación con o sin complicaciones, en la actualidad se interviene escasamente en el área de salud mental, y tampoco hay estudios que reporten la atención dada a los padres que atraviesan por el duelo perinatal (Kersting, Kroker, Schlicht, Baust y Wagner, 2011).

El apoyo brindado por los profesionales de la salud es un factor de gran importancia en el proceso de duelo de la familia, y su función se basa en actuar como guías que contribuyan a la toma de decisiones debido a la complejidad de la situación, además de proveer la información médica que soliciten los padres. La relación médico-paciente es de gran importancia, toda vez que a causa de diversas interacciones dañinas se pueden ocasionar consecuencias negativas a largo plazo en el duelo. En la misma línea, el abordaje de salud mental a cargo de profesionales de medicina y psiquiatría se fundamenta en la necesidad de brindar el asesoramiento necesario sobre la pérdida y el impacto que ésta tendrá en el futuro, realizar el seguimiento del estado emocional que acompaña al duelo, intervenir en la reducción de dicho malestar (Nikčević, Kuczmierczyk y Nicolaides, 2007) y, en caso de haber complicaciones de orden psiquiátrico, hacer uso de medicación (Lacasse y Cacciatore, 2014).

San Lazaro, Meaney, McNamara y O'Donoghoue (2017) realizaron una revisión sistemática de los efectos de las intervenciones en mujeres embarazadas que habían padecido el duelo perinatal. En sus conclusiones proponen intervenciones psicológicas luego de la pérdida como un elemento importante para lograr el bienestar emocional en un nuevo embarazo.

Para el manejo del duelo perinatal se han publicado algunos protocolos y guías centradas en ciertos aspectos ginecobstétricos, como el Late Intrauterine Fetal Death and Stillbirth. Green-top Guideline 55, del Real College of Obstetricians 
and Ginecologists (2010) o la Guía clínica de abordaje de duelo perinatal, de Duarte, Garcia y Mejías (2012). En lo relacionado con la salud mental, se han elaborado recomendaciones sobre la atención intrahospitalaria en los casos de muerte perinatal o neonatal; por lo tanto, es importante profundizar en los aspectos emocionales sobre los que se debe intervenir (Gold et al., 2007).

Por lo anteriormente expuesto, el objetivo de este trabajo fue evaluar el cumplimiento de las fases que componen la guía de atención de duelo perinatal en el ámbito hospitalario a través de una lista de chequeo y entrevista a las madres en duelo, teniendo en cuenta la percepción subjetiva de las mujeres respecto a cada fase de la atención brindada.

\section{MÉTODO}

Como un intento para ampliar la perspectiva epidemiológico-social (Muñoz, 2013), se realizó un estudio exploratorio, analítico, prospectivo, de tipo mixto en el que se combinan métodos cuantitativos y cualitativos para evaluar aspectos biomédicos y sociales, mismo que fue aprobado por el Comité de Ética en Investigación de la Fundación Universitaria Sanitas. La metodología cuantitativa tuvo en cuenta si se cumplen o no los primeros auxilios psicológicos, definidos por la OMS (2012) como "un modo práctico y humano para ayudar y apoyar a nuestros semejantes en graves situaciones de crisis $[. .$.$] pensado para personas que tienen la po-$ sibilidad de ayudar a otras que han experimentado un hecho extremadamente angustiante". La evaluación de la atención psicológica intrahospitalaria y psicológica de consulta externa se efectuó a través de un análisis de frecuencias. Los resultados relacionados con el cumplimiento de los componentes de la guía se presentan porcentualmente. El análisis de los datos cualitativos se hizo a través de la teoría fundamentada, en la cual se trabaja con entrevistas y basándose en la recolección y análisis sistemáticos de datos empíricos, sin ninguna teoría o hipótesis inicial (Hernández, 2014). Se establecieron categorías a priori y emergentes para la clasificación de la información.

Asimismo, se obtuvieron resultados del análisis mixto, en los que los datos cualitativos y cuan- titativos se cruzaron para determinar la percepción de las pacientes respecto al proceso de cumplimento de la guía. Todas las participantes firmaron un formato de consentimiento informado.

\section{Participantes}

Participaron nueve mujeres en duelo perinatal (Instituto Nacional de Salud Pública, 2016), asistentes a la institución, quienes acudían a consulta psicológica posterior a su egreso. Se excluyó a mujeres que solicitaron la interrupción voluntaria del embarazo.

\section{Instrumentos}

Guía de Atención en Salud Mental para Duelo Perinatal. Es este un instrumento no validado, diseñado en la Clínica Universitaria Colombia en 2009 y que forma parte de los elementos de abordaje del servicio de salud mental. Tiene en cuenta tres fases, la primera de las cuales es la emergencia obstétrica, en la que médicos y enfermeras llevan a cabo los primeros auxilios psicológicos (OMS, 2012) e interconsultan de manera prioritaria con el servicio de psicología. La segunda es la intervención de psicología, donde se hace la intervención en crisis. Aquí, el profesional de salud mental evalúa exhaustivamente cada factor que influye en el estado emocional de la persona, además de que establece y verifica un plan de seguimiento que dé respuesta inmediata a la situación de complejidad que enfrenta la persona (Slaikeu, 1996). Por último, la tercera fase corresponde al seguimiento posterior al egreso de la paciente del servicio de psicología, utilizándose para tal fin la terapia breve. Hewitt y Gantiva (2009) consideran esta fase como una estructura terapéutica cuya finalidad es establecer la manera en que los pacientes perciben su problemática para que asuman actitudes enfocadas al cambio; dicha terapia se enfoca en el presente y es de corta duración, con un máximo de cinco sesiones cuya finalidad es estabilizar a la paciente, evaluando para ello los posibles riesgos que pongan en peligro su salud física, emocional, social o espiritual.

Lista de chequeo. Para establecer el cumplimiento de la guía y las percepciones de las madres en duelo perinatal, se utilizó una lista de chequeo, 
que es un cuestionario que permite determinar la presencia, ausencia, estado o condición de la variable a observar (Álvarez, 2009). Incluye los componentes de la citada guía, donde la paciente refiere durante la entrevista si las actividades incluidas en la misma se llevaron a cabo durante el proceso de atención hospitalaria. Teniendo en cuenta que se trató de un estudio exploratorio, fue una lista y no una escala, por lo que se hizo un análisis de frecuencias, sin obtener medidas de consistencia interna.

Entrevista semiestructurada. A través de dicha entrevista se obtuvieron las percepciones de las pacientes. Estas entrevistas muestran una gran flexibilidad porque incorporan preguntas preestablecidas que pueden ajustarse a las personas entrevistadas (Díaz, Torruco, Martínez y Varela, 2013) (Tabla 1).

Tabla 1. Ejemplo de criterios utilizados en la lista de chequeo y la entrevista semiestructurada.

\begin{tabular}{|c|c|c|c|c|c|}
\hline \multicolumn{3}{|c|}{ LISTA DE CHEQUEO } & \multicolumn{3}{|c|}{ ENTREVISTA SEMI ESTRUCTURADA } \\
\hline $\begin{array}{c}\text { Primeros Auxilios } \\
\text { Psicológicos } \\
\text { (PAP) }\end{array}$ & $\begin{array}{c}\text { Psicología } \\
\text { intrahospitalaria }\end{array}$ & $\begin{array}{c}\text { Psicología } \\
\text { Consulta } \\
\text { Externa }\end{array}$ & $\begin{array}{c}\text { Primeros Auxilios } \\
\text { Psicológicos } \\
\text { (PAP) }\end{array}$ & $\begin{array}{c}\text { Psicología } \\
\text { Intrahospitalaria }\end{array}$ & $\begin{array}{c}\text { Psicología } \\
\text { Consulta } \\
\text { Externa }\end{array}$ \\
\hline Malas noticias & $\begin{array}{l}\text { Intervención en } \\
\text { crisis }\end{array}$ & $\begin{array}{l}\text { Número de citas } \\
\text { cumplidas }\end{array}$ & $\begin{array}{l}\text { ¿Quién le dio la } \\
\text { noticia? }\end{array}$ & $\begin{array}{l}\text { ¿La atención } \\
\text { psicológica fue } \\
\text { oportuna? }\end{array}$ & $\begin{array}{l}\text { Otros factores de } \\
\text { estrés asociados al } \\
\text { duelo }\end{array}$ \\
\hline Pudo preguntar & $\begin{array}{l}\text { Atención por } \\
\text { Psicología antes } \\
\text { del parto }\end{array}$ & $\begin{array}{l}\text { Atención en el } \\
\text { primer mes des- } \\
\text { pués de la pérdida }\end{array}$ & $\begin{array}{l}\text { ¿Estuvo alguien de } \\
\text { su familia acompa- } \\
\text { ñándola? }\end{array}$ & $\begin{array}{l}\text { ¿La atención de } \\
\text { Psicología fue } \\
\text { eficaz? }\end{array}$ & $\begin{array}{l}\text { Estrategias de } \\
\text { afrontamiento de } \\
\text { la paciente }\end{array}$ \\
\hline Ofrecieron apoyo & $\begin{array}{l}\text { Atención por } \\
\text { Psicología antes } \\
\text { del egreso }\end{array}$ & $\begin{array}{l}\text { Síntomas depre- } \\
\text { sivos }\end{array}$ & $\begin{array}{l}\text { ¿Cómo le dieron la } \\
\text { noticia? }\end{array}$ & $\begin{array}{l}\text { ¿Le ofreció estra- } \\
\text { tegias para enfren- } \\
\text { tar la situación? } \\
\text { Rituales }\end{array}$ & $\begin{array}{l}\text { Control de sínto- } \\
\text { mas depresivos y } \\
\text { ansiosos }\end{array}$ \\
\hline $\begin{array}{l}\text { Permitieron acom- } \\
\text { pañante }\end{array}$ & $\begin{array}{l}\text { Egresa con cita } \\
\text { programada }\end{array}$ & Síntomas ansiosos & $\begin{array}{l}\text { ¿Qué cambios } \\
\text { plantea cuando } \\
\text { tengan que dar este } \\
\text { tipo de noticias? }\end{array}$ & $\begin{array}{l}\text { ¿Le dio elementos } \\
\text { de apoyo emocio- } \\
\text { nal para el regreso } \\
\text { a casa? }\end{array}$ & $\begin{array}{l}\text { ¿Cómo se siente } \\
\text { con la intervención } \\
\text { psicológica? }\end{array}$ \\
\hline
\end{tabular}

\section{Procedimiento}

Tras identificar a las pacientes de acuerdo con los criterios de inclusión y exclusión, se seleccionaron aquellas que deseaban participar, haciéndoseles la intervención en salud mental siguiendo la guía de duelo perinatal. Se llevó a cabo el control posterior a su egreso, y por medio de la entrevista semiestructurada se facilitó al entrevistador una mejor comprensión del curso del duelo perinatal; además, la paciente pudo expresarse sobre el episodio, facilitando con ello la expresión emocional. Para obtener una información más veraz, se audiograbaron las sesiones para luego transcribirlas, lo que constituyó la base del análisis cualitativo. Asimismo, se aplicó la lista de chequeo revisando si se habían cumplido sus elementos: primeros auxilios psicológicos en la urgencia ginecobstétrica, atención intrahospitalaria por Psicología (lo que se confirmó con una nota en la historia clínica) y citas posteriores al egreso, lo que se verificó mediante la revisión del sistema de citas y la historia clínica. Cada elemento se valoró mediante cinco preguntas, respuestas de "sí" y "no", obteniéndose el porcentaje de cumplimiento de cada fase y el global.

\section{RESULTADOS}

Las pacientes, mujeres en duelo perinatal, reportaron edades de entre 20 y 35 años. La duración del embarazo en el que se encontraban al momento de la pérdida fue de 22 a 35 semanas.

A continuación, se presentan los resultados cuantitativos y cualitativos.

En lo relacionado con resultados los cuantitativos, el porcentaje de cumplimiento de la guía de duelo perinatal en cada fase fue como sigue: en primeros auxilios psicológicos las pacientes mostra- 
ron que se cumplió en $83 \%$; los ítems que consideraron incumplidos fueron los correspondientes al tiempo insuficiente del equipo médico al momento de dar la noticia (tres de las pacientes) y la manera inadecuada para informarles la pérdida del embarazo (en dos de ellas), generalmente por el empleo de un lenguaje muy técnico.

En la segunda fase, la atención intrahospitalaria por Psicología, se obtuvo un porcentaje de cumplimiento de $86 \%$. En este aspecto, las causas por las cuales no se cumplió dicha fase correspondieron a la demora de respuesta por parte de dicho servicio (tres mujeres no recibieron la atención inmediata después de recibida la noticia) y el nulo beneficio de la intervención. En la tercera, la consulta posterior al egreso, se obtuvo $100 \%$ de cumplimiento; de hecho, se dieron consultas a las nueve mujeres del estudio, a seis de ellas de manera inmediata y a las otras tres después de veinte días de su egreso de la clínica. En esta fase se realizaron las entrevistas semiestructuradas y las listas de chequeo. Finalmente, los resultados indican que el cumplimiento total de la guía fue de $86 \%$.

En cuanto a los resultados cualitativos de acuerdo con los relatos de las pacientes, estos se analizaron según cada una de las fases de la guía de duelo perinatal, para lo que se implementaron categorías presentes en los temas más frecuentes en cada fase, según lo obtenido en las entrevistas semiestructuradas. Para el análisis, se establecieron códigos con esas categorías, siguiendo para ello las recomendaciones de Saldaña (2009). En la Tabla 2 se presenta un ejemplo de tal codificación.

Tabla 2. Ejemplo de la codificación para el análisis cualitativo.

\begin{tabular}{|l|l|l|l|}
\hline \multicolumn{2}{|c|}{ CóDIGOS } & \multicolumn{1}{c|}{ PREGUNTAS RELACIONADAS } \\
\hline \multirow{3}{*}{ CÓDIGO 1 } & \multicolumn{1}{|c|}{ ETIQUETA } & \multicolumn{1}{c|}{ PAP } & ¿Cómo le dieron la noticia? \\
\cline { 2 - 3 } & DEFINICIÓN & $\begin{array}{l}\text { Momento en el que se informa la } \\
\text { iQuerte o la inminente muerte del feto. }\end{array}$ & $\begin{array}{l}\text { ¿Que la noticia? } \\
\text { ¿Qué sintió cuando le dieron la noticia? } \\
\text { ¿Cómo reaccionó cuando le dieron la noticia? }\end{array}$ \\
\cline { 2 - 4 } & SUBCATEGORÍA & Malas noticias. &
\end{tabular}

En la categoría de Primeros Auxilios Psicológicos (PAP) se planteó la subcategoría "Malas noticias" porque en esta etapa de la atención es necesario informar a las pacientes sobre las complicaciones en su estado de salud o en el del feto. Al realizar el proceso de análisis, es posible evidenciar otras subcategorías subyacentes: "sentir", "decir", "hacer"; las cuales corresponden a la forma en cómo las mujeres enfrentan su situación.

En la subcategoría "Sentir" las pacientes informaron dos tipos de reacciones: fisiológicas y emocionales. Por una parte, presentaron reacciones fisiológicas ajustadas a las percepciones adquiridas por medio de los sentidos que dan cuenta de un eventual riesgo. Las pacientes informaron no "escuchar" (en el sentido de audición) el latido de su bebé; "sentir" (tacto) sus movimientos; "ver" (visión) sangre o líquido y "oler" (olfato) un flujo fétido. «La ginecóloga me atiende, me hace tacto, me hace una ecografía y me mira a la bebé; me hace tacto y me huele. Dice: "El tacto huele a fétido". Me hacen un monitoreo con el ecógrafo, no ve señales de vida, se cerciora de que la bebé no tiene movimiento del corazoncito, que no hay nada. Luego dice la ginecóloga que no, que no hay movimientos de nada. Cuando ya descarta todas sus inquietudes, me dice:'Mamá, su bebé ya está muerta"». (Mujer 5, 25 años, 34 semanas). Vinculado a lo anterior, en cuanto a las reacciones emocionales, se evidencia una reacción de llanto acompañada de tristeza por el proceso que atraviesan, incluso acompañado de aceptación por "presentimientos". Otras pacientes comentan un estado de shock e incredulidad en el que refieren estar "guardando la esperanza" de que su bebé nazca vivo: "Yo guardaba la esperanza de que la niña estuviera viva, y no, ya la expulsé, y no, no dio señales de vida" (Mujer 3. 28 años, 20 semanas).

En la subcategoría "Decir" es posible establecer los factores "personas" y "noticia". Al referirse a "personas", las pacientes identifican dos actores al momento de recibir la noticia: el médico y su pareja y padre del bebé. Las mujeres encuentran en su pareja a su acompañante principal. En relación con el factor "noticia", hacen mención a lo referido por su médico sobre la complicación física que se relaciona con la muerte fetal o neonatal. 
En la subcategoría "Hacer" aparecen dos momentos claros en que la mujer se sitúa, un antes y un después de la noticia. Antes de conocer el fallecimiento, las mujeres dicen haber sido enviadas a procedimientos diagnósticos, como exámenes de laboratorio, ecografías, monitoreo fetal y otros con la finalidad de establecer el curso de la actual complicación, o la búsqueda de certeza sobre el óbito o la futura muerte neonatal. En relación a las acciones tomadas luego del nacimiento, las mujeres comentan el procedimiento realizado, y además la presencia o ausencia de dolor físico, para luego decidir si envían el cuerpo al servicio de patología para llevar a cabo la autopsia.

Continuando en la categoría de primeros auxilios (PAP), aparece otra subcategoría correspondiente a las redes de apoyo, alusivas a la necesidad de la persona de ponerse en contacto con sus familiares o amigos o buscar otro tipo de apoyo social (OMS, 2012). Las mujeres identifican principalmente a tres actores que brindan apoyo social durante el proceso. En primer lugar, sus familiares (con mayor frecuencia el padre del bebé), que otorgan principalmente un apoyo emocional e instrumental durante la estancia hospitalaria y que permanecen la mayor parte del tiempo en la clínica tras la noticia hasta el egreso de la paciente. Por otra parte, los profesionales de medicina y psicología y el apoyo informativo recibido: "La psicóloga me ayudó en el hecho de informarme qué podía hacer, porque obviamente yo ni sabía que iban a hacer con mi bebé: ¿botarlo? Ella me dijo lo que iban a hacer. El que más nos ayudó fue el primer médico, [que] nos dijo cómo pudo haber sido, las posibilidades, que no éramos los únicos, y la revisó y nos mostraba todo" (Mujer 3, 28 años, 20 semanas).

Finalmente, en la categoría PAP se halla la subcategoría de acciones enfocadas a la prevención y protección de otros daños, las cuales contribuyen a disminuir otros factores de estrés que pueden generar malestar emocional o experiencias traumáticas adicionales (National Child Traumatic Stress Network, 2006), y también el uso de técnicas basadas en evidencia, las cuales influyen positivamente en la elaboración del duelo. Durante la estancia hospitalaria, las mujeres identificaron las acciones tomadas por parte del equipo de salud enfocadas al control de la situación durante el nacimiento y luego de éste. Comentaron cómo se encontraba constituido el personal hospitalario, resaltando los profesionales de enfermería y ginecobstetricia. Algunas mujeres refieren cierto malestar por el número de estudiantes que asistían al momento de valoración y seguimiento médico: "Esperé y esperé, y ahí empezó todo el proceso. Entraban como estudiantes, iban, pasaban ¡y ya!... Después, los médicos empezaron a tratar de descartar lo que estaba pasando. Ya como al segundo día llegaron los médicos de alto riesgo y con un montón de estudiantes. Eso sí, llegaron y me miraron" (Mujer 9, 32 años, 24 semanas).

En términos generales, comentaron haberse sentido seguras con los procesos médicos realizados, aunque en ocasiones manifestaron percibir demora en la atención. Tras el nacimiento, las pacientes relataron el contacto que tuvieron con su bebé. Aunque no todas decidieron cargarlo o siquiera verlo, fue esa la recomendación dada por parte de los profesionales a cargo. Las experiencias de tal situación se basan completamente en la percepción sensitiva, táctil y visual: "La cargué. Es más, cuando me la pasaron aún estaba viva; entonces, cuando yo la cogí, lo que hice fue llamar a la enfermera y decirle que llamara a un médico, que ella estaba viva, y ella me decía que no, que no se podía hacer nada, que no iba a resistir. Le hablé a mi bebé, le conté todo lo que me había pasado. Yo la sentía ahogada; estaba moradita. La vi, la besé, la abracé, la tuve y luego ella se quedó ahí" (Mujer 1, 30 años, 21 semanas).

En la categoría de "Atención psicológica intrahospitalaria" se tiene implementada la intervención en crisis por parte del profesional de salud mental, la cual se enfoca en el psicodiagnóstico y acompañamiento para el soporte emocional. No todas las mujeres recibieron acompañamiento durante la etapa de crisis en razón de que algunas no lo consideraron necesario. Las que lo recibieron expresaron que dicho apoyo tuvo resultados positivos y refirieron estar mejor preparadas para afrontar el suceso luego del egreso hospitalario. Una paciente se mostró reactiva frente al proceso. Expresó: «Durante los tres días que estuve hospitalizada [...] yo estaba como errada; no quería escuchar a nadie, no quería que nadie me diera condolencias y yo estaba muy mal. Sentía que todo el mundo venía a decirme: “¡Ay, qué pesar! 
Se murió su bebé", y como que tiene uno la llaga abierta y le echan sal. Eso entonces era lo que yo sentía» (Mujer 2, 35 años, 39 semanas).

Aquellas que no fueron atendidas de inmediato debido a que ciertos recursos no estaban disponibles los fines de semana, por el horario nocturno durante la urgencia o por la elevada amenaza a la vida materna o fetal que requería proceder con prontitud, expresaron el deseo de haber dispuesto de dicha asistencia en salud mental en su momento. "Me hubiera gustado que desde ahí hubiera estado alguien, independientemente de mi familia, porque necesitaba ese apoyo moral, pero nunca [lo hubo] Pero sí me hubiera gustado que alguien estuviera ahí [para] ayudarme a tomar decisiones más correctas “ (Mujer 5, 25 años, 34 semanas).

Otra fase de la atención por Psicología es la relacionada con el seguimiento, cuya finalidad es brindar soporte emocional y acompañamiento en cada parte del proceso de duelo; además, es aquí donde se establece la necesidad de continuar en consulta externa para llevar a cabo la intervención y prevenir complicaciones en la salud mental. Las nueve mujeres recibieron atención psicológica previa a su egreso hospitalario.

Finalmente, respecto a la atención psicológica posterior al egreso, las pacientes comentaron su percepción acerca de la atención recibida, las instalaciones y el actuar profesional. Fue posible evidenciar ciertas barreras para asistir a consulta externa para continuar la intervención psicológica. Una de las pacientes que acudió después de veinte días de su egreso hospitalario comentó: "No deseo recordar lo sucedido o volver a la clínica" (Mujer 2, 35 años, 39 semanas).

Como resultado, las pacientes opinaron sobre el trato recibido por parte de los profesionales de salud; por una parte, algunas tuvieron dificultades en la comunicación y otras no lograron identificar errores durante el proceso. "A mí me daba mucha rabia que unos dijeran una cosa y otros [...] otra cosa. Obviamente, había unos casos en los que estaban descartando situaciones. Supongo que estaban haciendo eso: descartando un diagnóstico, y así..." (Mujer 2, 35 años, 39 semanas). Sin embargo, la mayor parte de las mujeres que no hallaron dificultades durante la atención por parte de los profesionales son las que fueron intervenidas en los quince días siguientes a su egreso de la clínica.
En el marco del análisis mixto de la información, se correlacionan los datos cuantitativos que muestran dificultades para el cumplimiento total de los criterios establecidos, en conjunto con los resultados cualitativos que contienen las percepciones de las mujeres respecto a la atención brindada en cada fase.

Así, en la fase PAP las pacientes puntuaron bajo en las preguntas y expresaron que los médicos se centraban en la atención médica, las complicaciones o la demora en el trabajo de parto. Otro aspecto que tuvo una baja puntuación fue el poco tiempo destinado a responder dudas; de hecho, las inquietudes que tenían las mujeres no fueron resueltas en su totalidad.

En cuanto a la fase de atención psicológica intrahospitalaria, algunas de ellas no recibieron atención en crisis debido a que la urgencia ginecológica no permitió el manejo por Psicología. En una de las pacientes no fue posible encontrar la razón por la cual no contactó el servicio en ese momento. En la misma línea, dos pacientes refirieron que la intervención por Psicología no tuvo efecto alguno en su estado emocional.

Respecto a la fase final de la consulta externa por Psicología, a la totalidad de las pacientes se les hizo un seguimiento posterior a su egreso hospitalario. El estudio reveló algunas barreras para continuar el proceso psicológico en consulta externa, pues algunas pacientes señalaron sentir malestar por los recuerdos sobre lo vivido, evitaron asistir a la clínica o mostraron resistencia a hablar sobre lo sucedido.

\section{DISCUSIÓN}

En esta investigación se logró establecer un cumplimiento promedio de $83 \%$ de los criterios propuestos en la guía de duelo perinatal. Los factores que contribuyeron a que no se lograra su cumplimiento cabal tienen que ver con dificultades en el manejo de los primeros auxilios psicológicos a todas las pacientes en duelo, las demoras en la atención $\mathrm{y}$, en la consulta posterior al egreso, la inasistencia de la paciente en duelo.

En el caso de los primeros auxilios psicológicos, algunas pacientes manifestaron una relación distante con su médico tratante, en especial al momento 
de darles la noticia. Por ende, se debe procurar un entrenamiento dirigido a dichos profesionales enfocado en la comunicación con las pacientes, y haciendo uso de herramientas validadas, como es el caso del protocolo EPICEE, el cual permite al médico un manejo metódico al momento de dar una mala noticia (Buckman, 1992); además, es indispensable fomentar en ellos el contacto asertivo, libre de juicios y procurando evitar el uso de terminología médica. Adicionalmente, el profesional de salud debe saber contener las reacciones físicas y emocionales propias del estado de crisis, y canalizar a la persona al servicio de psicología de manera oportuna.

Molina (2016) confirma los hallazgos del presente estudio al subrayar el insuficiente entrenamiento del profesional de medicina en lo concerniente a la comunicación que establece con sus pacientes, lo cual a su vez puede estar vinculado a la formación académica con la que cuenta, ya que los contenidos curriculares correspondientes a esa temática no son uniformes en todas las instituciones universitarias (Rivera, Veres, Rego y Túñez, 2016), lo que fomenta actuar de manera subjetiva y a partir de experiencias personales previas, por lo cual es importante que el profesional se capacite para obtener las habilidades esperadas para abordar situaciones de alta complejidad.

Así, gran parte de mujeres refirieron haber percibido demoras en la atención dada por los profesionales de salud, factor que afecta la relación médico-paciente, por lo que es importante comunicar a las mujeres el motivo del retraso a fin de reducir la incertidumbre ante el proceso, lo que no implica que esta única estrategia vaya a generar una satisfacción inmediata en la totalidad de las pacientes; como lo señalan Salvador, Millán, Téllez, Pérez y Oliver (2013), el factor de espera se encuentra relacionado con la atención integral recibida, por lo que es necesario realizar ajustes de fondo en la organización de las instituciones de salud con la finalidad de procurar una mayor calidad en el servicio que se ofrece a las pacientes.

Durante la estancia hospitalaria, los profesionales de salud se convierten en una de las principales redes de apoyo; en efecto, el equipo multidisciplinar desempeña una importante función para la paciente, por lo que se debe asegurar un contexto que permita una interacción empática y amable (González, 2006), factor que se considera esencial en cada fase de la guía propuesta.

Al considerar el papel de los profesionales de salud, se debe procurar evitar otras situaciones que generen traumas en las mujeres. Por ejemplo, los relatos evidencian cierto malestar relacionado con las visitas que hacían los médicos en compañía de grandes grupos de estudiantes. Es aquí donde es importante considerar planteamientos como el expuesto por Orellana, Olmos, Rojas y Rebolledo (2011), relacionados con la sobreexposición del paciente en los hospitales docentes por el número de estudiantes que se hallan en proceso de práctica, lo que produce el agotamiento de los pacientes y la pérdida de la intimidad, por lo que los mencionados autores recomiendan hacer uso de formatos de consentimiento informado verbal siempre que se lleve a cabo un procedimiento médico en compañía de alumnos como parte de la labor docente.

El rol de la clínica en cuanto a la prevención de daños mayores se enfoca en la política de humanización plasmada en las recomendaciones de la OMS (2015b) para el parto humanizado, destinadas a garantizar el respeto a los derechos humanos, la dignidad de las mujeres y el mantenimiento de normas éticas y de seguridad en la práctica clínica. Entre esas recomendaciones sobresale el alivio del dolor y el acompañamiento permanente durante el trabajo de parto, así como el contacto entre los padres y el hijo fallecido, por lo que es necesario preguntar a aquellos sobre su deseo de verlo y sostenerlo en los brazos, ya que muchos tienden a manifestar ideas relacionadas con el arrepentimiento por no haberlo hecho; sin embargo, siempre es una decisión que deben tomar los padres, sin sentirse obligados a ello (Neril, 2012).

Al hacer referencia a la intervención psicológica durante la estancia hospitalaria, varias mujeres no recibieron acompañamiento por Psicología durante la etapa de crisis debido a la intervención médica inmediata necesaria para salvar la vida de la madre. Por esta razón, se debe procurar la intervención psicológica a la mayor brevedad posible para solventar dicha falta. Geller et al. (2010) analizó el tratamiento que las mujeres recibían luego de una pérdida, hallando que, en general, no incluye el apoyo emocional y psicosocial, y cuando existe, las pacientes refieren insuficiencias. La propuesta 
de Hutti et al. (2018) es valiosa en el caso de este tipo de pacientes no solo por la valoración mediante instrumentos, sino por el tiempo que proponen después de la pérdida. Por lo anterior, es indispensable continuar dicha atención, y de esta manera brindar apoyo teniendo en cuenta las reacciones inmediatas del duelo, tan potencialmente traumáticas para la mujer (Engelhard, van den Hout y Arntz, 2001).

En el presente estudio se encontró que una mujer no deseaba recibir atención psicológica, por lo cual es importante enfatizar que la paciente es la única que puede decidir si desea continuar o no con dicho acompañamiento.

Finalmente, al realizar el seguimiento posterior al egreso de estas pacientes aparecen diversas barreras que pueden influir en sus percepciones sobre la atención por Psicología, considerándola en algunos casos innecesaria, intimidante o incluso alarmante, sobre todo en el caso de quienes manifiestan poder lograr en el futuro un apropiado control emocional sin necesidad de apoyo, así como de quienes manifiestan evitación emocional y experiencial, por lo que es necesario realizar acercamientos oportunos luego de un mes de la atención hospitalaria. Como indican Lacasta, Limonero, García y Barahona (2014) en su estudio sobre la atención que brindan grupos enfocados en el duelo, el primer seguimiento generalmente lo realizan al mes, luego a los tres meses, o bien periódicamente a partir del mes de ocurrido el evento. En el mismo sentido, San Lazaro et al. (2017) señalan la importancia de llevar a cabo intervenciones después de un aborto como un elemento importante para brindar apoyo emocional en futuros embarazos. Por ello, se recomienda que las pacientes reciban esa atención en un sitio donde se sientan seguras, pues la estimulación pro- pia de la clínica puede desencadenar una elevada reacción emocional; por consiguiente, algunos profesionales están incursionando en el campo de la intervención cognitivo-conductual por medio de herramientas virtuales (Eisma et al., 2015), e incluso por otros medios, como el seguimiento telefónico, lo que puede reducir los síntomas depresivos (Neugebauer et al., 2007). Así, es importante examinar qué opciones se le pueden ofrecer a las pacientes para que puedan ser atendidas en aquellos casos en que se les dificulta acercarse a la clínica.

Durante la investigación se encontraron limitaciones respecto al tamaño de la muestra y el tipo de muestreo para el análisis cuantitativo; sin embargo, al recuperar el relato de las pacientes se dispone de información cualitativa valiosa, a lo que se suma el análisis mixto, que permite correlacionar resultados.

En conclusión, al hacer referencia a otros interrogantes que surgen a raíz del presente estudio, se plantea la necesidad de conocer las barreras que puede encarar el profesional de salud, mismas que afectan negativamente su acercamiento a las pacientes en duelo.

La guía de atención en duelo perinatal se constituye como un instrumento útil para el personal de salud, tal como lo plantean las pacientes incluidas en este trabajo. Respecto a lo concerniente a la labor del servicio de Psicología, se constata que es necesario en todo el proceso, ya sea intrahospitalario o externo, porque ayuda al bienestar emocional de las pacientes. Por último, el cumplimiento de la guía permite mejorar procesos de retroalimentación y a la vez brindar mayor calidad y calidez en la atención durante una pérdida perinatal.

\section{REFERENCIAS}

Álvarez, F., y Álvarez, A. (2009). Auditoría médica y epidemiología. Bogotá: Ecoe Ediciones.

American Psychiatric Association (2013). Diagnostic and Statistic Manual of Mental Disorders (5ª ed.). Washington, DC: APA.

Bello, R. (2016). Las experiencias de las mujeres durante el embarazo a partir del análisis del discurso. Revista de Investigación en Mujer, Salud y Sociedad, MUSAS, 1(1), 63-81.

Boelen, P., Keijser, J., van den Hout, M. y Van den Bout, J. (2007). Treatment of complicated grief: A comparison between cognitive-behavioral therapy and supportive counseling. Journal of Consulting and Clinical Psychology, 75(2), 277-284. doi: 10.1037/0022-006X.75.2.277. 
Buckman, R. (1992). How to break bad news. A guide for health care professionals. Baltimore, MD: John Hopkins.

Departamento Administrativo Nacional de Estadística (2016a). Encuesta Nacional de Demografía y Salud: Defunciones de menores de un año por grupos de edad y sexo, según departamento de año 2015. Recuperado de http://www.dane.gov.co/ index.php/esp/poblacion-y-demografia/nacimientos-y-defunciones/118-demograficas/estadisticas-vitales.

Departamento Administrativo Nacional de Estadística (2016b). Encuesta Nacional de Demografía y Salud: Defunciones fetales por área y sexo, según departamento de ocurrencia, total nación año 2015. Recuperado de http://www.dane.gov.co/index. $\mathrm{php} / \mathrm{esp} /$ poblacion-y-demografia/nacimientos-y-defunciones/118-demograficas/estadisticas-vitales/5579-defunciones-fetales-2014-preliminar.

Díaz B., L., Torruco G., U., Martínez H., M. y Varela R., M. (2013). La entrevista, recurso flexible y dinámico. Investigación en Educación Médica, 2(7), 162-167.

Duarte, L., García, S. y Mejías M., C. (2012). Guía clínica de abordaje en duelo perinatal. Madrid: Bubok Publishing.

Eisma, M., Boelen, P., van den Bout, J., Stroebe, W., Schut, H., Lancee, J. y Stroebe, M. (2015). Internet-based exposure and behavioral activation for complicated grief and rumination: A randomized controlled trial. Behavior Therapy, 46(6), 729748. doi: 10.1016/j.beth.2015.05.007.

Engelhard, I., van den Hout, M. y Arntz, A. (2001). Posttraumatic stress disorder after pregnancy loss. General Hospital Psychiatry, 23(2), 62-66. doi: 10.1016/S0163-8343(01)00124-4.

Fescina, R., De Mucio, B., Ortiz, E., Jarquín, D. (2012). Guías para la atención de las principales emergencias obstétricas. Lima: Consorcio Latinoamericano contra el Aborto Inseguro (CLACAI). Recuperado de http://www.clacaidigital.info:8080/xmlui/ bitstream/handle/123456789/782/CLAP1594.pdf?sequence=1yisAllowed=y.

García, C. y Yagüe, A. (1999). Duelo perinatal. Revista de Enfermería, 10, 17-20. Recuperado de https://www.uclm.es/ab/enfermeria/revista/numero\%2010/revista10.pdf.

Geller, P., Psaros, C. y Kornfield, S. (2010). Satisfaction with pregnancy loss aftercare: are women getting what they want? Archives of Women's Mental Health, 13(2), 111-124.

Gold, K., Dalton, V. y Schwenk, T. (2007). Hospital care for parents after perinatal death. Obstetrics \& Gynecology, 109(5), 11561166. doi: 10.1097/01.AOG.0000259317.55726.df.

González, R. (2006). La relación equipo de salud-paciente-familiar. Revista Cubana de Salud Pública, 32(3) Recuperado de http://scielo.sld.cu/scielo.php?script=sci_arttextypid=S0864-34662006000300011ylng=esytlng=es.

Hernández, R. (2014). La investigación cualitativa a través de entrevistas: su análisis mediante la teoría fundamentada. Cuestiones Pedagógicas, 23, 187-210.

Hewitt, N. y Gantiva, C. (2009). La terapia breve: Una alternativa de intervención psicológica efectiva. Avances En Psicologia Latinoamericana, 27(1), 165-176.

Hutti, M.H., Myers, J.A., Hall, L.A., Polivka, B.J., White, S., Hill, J., Grisanti, M., Hayden, J., y Kloenne, E. (2018). Predicting need for follow-up due to severe anxiety and depression symptoms after perinatal loss. Journal of Obstetric, Gynecoly and Neonatal Nursing, Jan 31. Recuperado de pii: S0884-2175(18)30003-0. doi: 10.1016/j.jogn.2018.01.003.

Instituto Nacional de Salud Pública (2016). Protocolo de vigilancia en salud pública, mortalidad perinatal y neonatal tardía 2016. México: INSP. Recuperado de http://www.ins.gov.co/lineas-de-accion/Subdireccion Vigilancia/sivigila/Protocolos\%20SIVIGILA/PRO\%20Mortalidad \%20perinatal\%20y\%20neonatal.pdf.

Kersting, A., Kroker, K., Schlicht, S., Baust, K. y Wagner, B. (2011). Efficacy of cognitive behavioral internet-based therapy in parents after the loss of a child during pregnancy: Pilot data from a randomized controlled trial. Archives of Women's Mental Health, 14(6), 465-477. doi: 10.1007/s00737-011-0240-4.

Lacasse, J. y Cacciatore, J. (2014). Prescribing of psychiatric medication to bereaved parents following perinatal/neonatal death: an observational study. Death Studies, 38(9), 589-596.

Lacasta, M., Limonero, J., García, A. y Barahonad, H. (2014). La atención al duelo en cuidados paliativos. Análisis de los servicios prestados en España. Medicina Paliativa, 23(4), 192-198.

Li, J., Precht, D., Mortensen, P. y Olsen, J. (2003). Mortality in parents after death of a child in denmark: A nationwide follow-up study. The Lancet, 361, 363-367. doi: 10.1016/S0140-6736(03)12387-2.

Medina, J. (2015). Trauma psíquico. Madrid: Ediciones Paraninfo, S.A.

Ministerio de Salud de Colombia (1993). Resolución 008430 del 4 de Octubre de 1993. Bogotá: Ministerio de Salud.

Molina, M. (2016). Propuesta de Intervención en habilidades de comunicación en médicos. Trabajo inédito de grado. Elche (España): Universidad Miguel Hernández.

Muñoz, C. (2013). Métodos mixtos: una aproximación a sus ventajas y limitaciones en la investigación de sistemas y servicios de salud. Revista Chilena de Salud Pública, 17(3), 218-223.

National Child Traumatic Stress Network (2006). Primeros Auxilios Psicológicos: Guía de Operaciones Prácticas (2 ${ }^{\mathrm{a}}$ ed.). Recuperado de http://www.nctsn.org/sites/default/files/pfa/spanish/1-primeros_auxilios_book_final_comp_guide.pdf.

Neril, L. (2012). Intervención paliativa desde la psicología perinatal. Revista del Hospital Materno-Infantil Ramón Sardá, 31(1), $18-21$. 
Neugebauer. R., Kline, J., Bleiberg, K., Baxi, L., Markowitz, J., Rosing, M. y Keith, J. (2007). Preliminary open trial of interpersonal counseling for subsyndromal depression following miscarriage. Depress Anxiety, 24(3), 219-222.

Nikčević, A., Kuczmierczyk, A. y Nicolaides, K. (2007). The influence of medical and psychological interventions on women's distress after miscarriage. Journal of Psychosomatic Research, 63(3), 283-290. doi: 10.1016/j.jpsychores.2007.04.004.

Orellana P., C., Olmos O., J., Rojas D.V., J. y Rebolledo G., E. (2011). Sobreexposición de los pacientes en hospitales docentes: la experiencia en un servicio de medicina. Persona y Bioética, 15(1), 78-89.

Organización Mundial de la Salud (2015a). Mortalidad materna. Ginebra: OMS. Recuperado de http://www.who.int/mediacentre/ factsheets/fs348/es/.

Organización Mundial de la Salud (2015b). Recomendaciones de la OMS para la conducción del trabajo de parto. Ginebra: OMS. Recuperado de http://www.who.int/topics/maternal_health/directrices_oMs_parto_es.pdf.

Organización Mundial de la Salud (2012). Primera ayuda psicológica: Guía para trabajadores de campo. Ginebra: OMS. Recuperado de http://apps.who.int/iris/bitstream/10665/44837/1/9789243548203_spa.pdf.

Organización Panamericana de la Salud (2011). Salud mental perinatal. Washington, D.C.: OPS.

Organización Panamericana de la Salud (2016). Guía de intervención humanitaria mhGAP (GIH-mhGAP). El manejo clínico de los trastornos mentales, neurológicos y por uso de sustancias en las emergencias humanitarias. Washington, D.C.: OPS. Recuperado de http://iris.paho.org/xmlui/handle/123456789/28418.

Oviedo S., S., Urdaneta C., E., Parra F., F. y Marquina V., M. (2009). Duelo materno por muerte perinatal. Revista Mexicana de Pediatría, 76(5), 215-219.

Rabelo, J. (2010). Primeros auxilios psicológicos e intervención en crisis desde el trabajo social. Documentos de Trabajo Social, 47, 121-133.

Rivera, A., Veres, E., Rego, F. y Túñez, J. (2016). Análisis de la formación en comunicación y la relación médico-paciente en los grados de medicina en España. Index. Comunicación, 6(1). 27-51. Recuperado de http://journals.sfu.ca/indexcomunicacion/index.php/indexcomunicacion/article/view/206/193.

Royal College of Obstetricians and Gynaecologists (2010). Late intrauterine fetal death and stillbirth. Green-top Guideline, 55. Recuperado de https://www.rcog.org.uk/globalassets/documents/guidelines/gtg_55.pdf.

Saldaña, J. (2009). The coding manual for qualitative researchers. Thowsand Oaks: Sage.

Salvador, F., Millán, J., Tellez, C., Pérez, C. y Oliver, C. (2013). Implantación de un plan de calidad en un servicio de urgencias y su impacto en la satisfacción del paciente. Emergencias. Revista de la Sociedad Española de Medicina de Urgencias y Emergencias, 25(3), 163-170.

San Lazaro C., I., Meaney, S., McNamara, K. y O’Donoghue, K. (2017). Psychological and support interventions to reduce levels of stress, anxiety or depression on women's subsequent pregnancy with a history of miscarriage: an empty systematic review. British Medical Journal, 7(9). Recuperado de http://www.lenus.ie/hse/bitstream/10147/622592/1/Psychological.pdfs.

Sanz, J., García, I. y Carbajo, E. (2014). Tratamiento del duelo en salud mental: una experiencia grupal. Revista de Psicoterapia, 25(99), 115-133.

Slaikeu, K. (1996). Intervención en crisis, manual para práctica e investigación (2ª ed.). México: El Manual Moderno. 